\title{
SHANNON ENTROPY AND CHARACTERIZATION OF NADARAJAH AND HAGHIGHI DISTRIBUTION BASED ON GENERALIZED ORDER STATISTICS
}

\section{J. S. KHAN and ARTI SHARMA}

Department of Statistics and Operations Research

Aligarh Muslim University

Aligarh-202 002

India

e-mail: jahangirskhan@gmail.com

arti414gc@gmail.com

\begin{abstract}
In this paper, we have derived the exact expression for the Shannon entropy of Nadarajah and Haghighi (NH) distribution based on generalized order statistics. Characterization of $\mathrm{NH}$ distribution is discussed by using the recurrence relation based on single and product moments of generalized order statistics. And we have obtained the exact and explicit expression for single and product moment of generalized order statistics in terms of Gauss hypergeometric function and Kampé de Fériet series from NH distribution. Further, we have discussed the results of ordinary order statistics, progressive type II censoring and record values.
\end{abstract}

2010 Mathematics Subject Classification: 62G30.

Keywords and phrases: Shannon entropy, generalized order statistics, order statistics, progressive type II censoring, record values, characterization, NH (Nadarajah and Haghighi) distribution.

Received January 29, 2018; Revised February 14, 2018

(C) 2018 Scientific Advances Publishers 


\section{Introduction}

The concept of entropy was originated by Shannon [24] in the nineteenth century. It is a mathematical measure of information which measures the average reduction of uncertainty of $X$. The Shannon entropy for a continuous random variable $X$ with probability density function $f(x)$ is defined as

$$
H(X)=-\int_{-\infty}^{+\infty} f_{X}(x) \ln f_{X}(x) d x
$$

The entropy of order sequences and order statistics was discussed by Wong and Chen [26]. Madadi and Tata [17, 18, 19] have deduced the Shannon and Rényi information in the record data. Afhami and Madadi [1] have obtained the exact form of Shannon entropy based on generalized order statistics (gos) from Pareto-type distribution. Mahmoud and Ghafour [20] have obtained the Shannon information for generalized Feller-Pareto family.

Ordered random variables are widely used in reliability theory and life testing. However, they can be considered as special cases of gos which has been introduced and extensively studied by Kamps [7]. It enables a unified approach to several models of ordered random variables such as ordinary order statistics, record values, sequential order statistics and progressively type II censored statistics. Let $X_{1}, X_{2}, \ldots, X_{n}$ be a sequence of independent and identically distributed (iid) random variables with the $c d f \quad F(x)$ and the $p d f \quad f(x)$. Let $k \in \mathbb{N}$, $\tilde{m}=\left(m_{1}, m_{2}, \ldots, m_{n-1}\right) \in \mathbb{R}^{n-1}, \gamma_{j}=k+n-j+\sum_{i=j}^{n-1} m_{i}>0 . \quad$ Then $X(1, n, \tilde{m}, k), X(2, n, \tilde{m}, k), \ldots, X(n, n, \tilde{m}, k)$ are said to be gos if their joint $p d f$ is given by

$$
k\left(\prod_{j=1}^{n-1} \gamma_{j}\right)\left(\prod_{i=1}^{n-1}\left[\bar{F}\left(x_{i}\right)\right]^{m_{i}} f\left(x_{i}\right)\right)\left[\bar{F}\left(x_{n}\right)\right]^{k-1} f\left(x_{n}\right),
$$

on the cone $F^{-1}(0+)<x_{1} \leq x_{2} \leq \ldots \leq x_{n}<F^{-1}(1)$ of $\mathbb{R}^{n}$, where $\bar{F}(x)=1-F(x)$ denotes the survival function. 
Here we may consider two cases:

Case I: $\quad \gamma_{i} \neq \gamma_{j} ; \forall i \neq j=1,2 \ldots, n-1, \quad$ i.e., $\quad \gamma_{i}$ 's are pairwise different.

In this case, the $p d f$ of $X(r, n, \tilde{m}, k)$ is given by (Kamps and Cramer [9])

$$
f_{X(r, n, \tilde{m}, k)}(x)=c_{r-1} \sum_{i=1}^{r} a_{i}(r)[\bar{F}(x)]^{\gamma_{i}} \frac{f(x)}{\bar{F}(x)}, \quad-\infty \leq x \leq \infty,
$$

and the joint $p d f$ of $X(r, n, \tilde{m}, k)$ and $X(s, n, \tilde{m}, k)$, where $1 \leq r<s \leq n$, is given by (Kamps and Cramer [9])

$$
\begin{aligned}
f_{X(r, n, \tilde{m}, k), X(s, n, \tilde{m}, k)}(x, y)= & c_{s-1}\left[\sum_{t=r+1}^{s} a_{t}^{(r)}(s)\left[\frac{\bar{F}(y)}{\bar{F}(x)}\right]^{\gamma_{t}}\right]\left[\sum_{i=1}^{r} a_{i}(r)[\bar{F}(x)]^{\gamma_{i}}\right] \\
& \times \frac{f(x)}{\bar{F}(x)} \frac{f(y)}{\bar{F}(y)},-\infty \leq x<y \leq \infty
\end{aligned}
$$

where

$$
\begin{aligned}
& c_{r-1}=\prod_{i=1}^{r} \gamma_{i}, \\
& a_{i}(r)=\prod_{\substack{j=1 \\
j \neq i}}^{r} \frac{1}{\left(\gamma_{j}-\gamma_{i}\right)}, \gamma_{j} \neq \gamma_{i}, 1 \leq i \leq r \leq n,
\end{aligned}
$$

and

$$
a_{t}^{(r)}(s)=\prod_{\substack{t=r+1 \\ t \neq i}}^{s} \frac{1}{\left(\gamma_{t}-\gamma_{i}\right)}, \gamma_{t} \neq \gamma_{i}, r+1 \leq t \leq s \leq n .
$$

Case II: $m_{1}=m_{2}=\ldots=m_{n-1}=m$ (say), $i=1,2, \ldots, n-1$.

In this case, the $p d f$ of $r$-th gos is given by (Kamps [7])

$$
f_{X(r, n, m, k)}(x)=\frac{c_{r-1}}{(r-1) !}[\bar{F}(x)]^{\gamma_{r}-1} g_{m}^{r-1}(F(x)) f(x), \quad-\infty \leq x \leq \infty,
$$


and the joint $p d f$ of $X(r, n, m, k)$ and $X(s, n, m, k), 1 \leq r<s \leq n$, is given by

$$
\begin{aligned}
f_{X(r, n, m, k), X(s, n, m, k)}(x, y) & \\
= & \frac{c_{s-1}}{(r-1) !(s-r-1) !}[\bar{F}(x)]^{m} g_{m}^{r-1}(F(x)) \\
& \times\left[h_{m}(F(y))-h_{m}(F(x))\right]^{s-r-1}[\bar{F}(y)]^{\gamma_{s}-1} f(x) f(y), \\
& -\infty \leq x<y \leq \infty,
\end{aligned}
$$

where

$$
h_{m}(x)=\left\{\begin{array}{lc}
-\frac{(1-x)^{m+1}}{m+1}, & m \neq-1, \\
-\ln (1-x), & m=-1,
\end{array}\right.
$$

and

$$
g_{m}(x)=h_{m}(x)-h_{m}(0), \quad x \in(0,1) .
$$

Choosing the parameters appropriately, models such as ordinary order statistics $\left(m=0, k=1\right.$, i.e., $\left.\gamma_{i}=n-i+1\right), k$-th record value $(m=-1$, $k \in \mathbb{N}$, i.e., $\left.\gamma_{i}=k\right)$, sequential order statistics $\left[\gamma_{i}=(n-i+1) \beta_{i} ; \beta_{1}, \beta_{2}, \ldots\right.$, $\left.\beta_{n}>0\right]$, order statistics with non-integral sample size $\left[\gamma_{i}=(\beta-i+1)\right.$; $\beta>0]$, Pfeifer record values $\left(\gamma_{i}=\beta_{i} ; \beta_{1}, \beta_{2}, \ldots, \beta_{n}>0\right)$ and progressive type II censored order statistics $\left(n, m \in \mathbb{N}, n=m+\sum_{j=1}^{m} R_{j}, \quad R_{j} \in \mathbb{N}_{0}\right.$ and $\left.\gamma_{j}=n-\sum_{\nu=1}^{j-1} R_{\nu}-j+1,1 \leq j \leq m\right)$ can be seen as particular cases of gos. Several authors discussed the concept of gos, for example, Hwang and Lin [6], Ahsanullah [3], Ahmad [2], Khan and Kumar [11], Khan and Khan [10], Kulshrestha et al. [12]. 
Kamps [8] has described the importance of recurrence relations of order statistics in characterization. Su and Huang [25] has discussed the characterization based on conditional expectations. Arslan [4] characterized the uniform distribution by generalized order statistics. Generalized order statistics from Kumaraswamy distribution and its characterization was discussed by Kumar [13].

Recently, Nadarajah and Haghighi [22] have introduced a new extension of exponential distribution as an alternative to the gamma, Weibull and the exponentiated exponential distribution with $p d f$

$$
f(x: \sigma, \theta)=\frac{\theta}{\sigma}\left(1+\frac{x}{\sigma}\right)^{\theta-1} \exp \left[1-\left(1+\frac{x}{\sigma}\right)^{\theta}\right], x>0,
$$

where $\sigma>0$ and $\theta>0$ are the scale and shape parameters, respectively. Lemonte [16] gave a new generalization of $\mathrm{NH}$ distribution through exponentiation of (1.7). The corresponding $c d f$ of the $\mathrm{NH}$ distribution is

$$
\begin{gathered}
F(x: \sigma, \theta)=1-\exp \left[1-\left(1+\frac{x}{\sigma}\right)^{\theta}\right], x>0, \\
\bar{F}(x)=\frac{\sigma}{\theta}\left(1+\frac{x}{\sigma}\right)^{1-\theta} f(x), x>0,
\end{gathered}
$$

$\mathrm{NH}$ distribution can be used to model data that have their mode fixed at zero. At $\theta=1, \mathrm{NH}$ distribution reduces to the one parameter exponential distribution.

MirMostafaee et al. [21] studied the record values from $\mathrm{NH}$ distribution. Some results on order statistics has been done for extended exponential distribution by Kumar et al. [15]. And Kumar and Dey [14] derived relations for moments of generalized order statistics from extended exponential distribution. Selim [23] has discussed the estimation and prediction for Nadarajah-Haghighi distribution based on record values. 
The rest of the paper is organized as follows. In Section 2, we have obtained the Shannon entropy of $\mathrm{NH}$ distribution based on gos. In Section 3, characterization based on recurrence relation of the single moment of gos from $\mathrm{NH}$ distribution is given. In Section 4, we have characterized $\mathrm{NH}$ distribution based on recurrence relation of the product moment of gos. In Section 5, we have computed the mean and variance of the order statistics and gos from $\mathrm{NH}$ distribution. We have made some conclusions about the NH distribution in Section 6.

\section{Shannon Entropy of NH Distribution Based on Generalized Order Statistics}

In this section, we have obtained the exact and analytical expression of the Shannon entropy of NH distribution based on gos. Let $\mathbf{X}(n, \tilde{m}, k)$ $=(X(1, n, \tilde{m}, k), \ldots, X(n, n, \tilde{m}, k))$ be a vector of gos, then

$$
\begin{aligned}
f_{\mathbf{X}(n, \tilde{m}, k)}(\mathbf{x})= & k\left(\prod_{j=1}^{n-1} \gamma_{j}\right)\left(\prod_{t=1}^{n-1}\left\{\left[\exp \left(1-\left(1+x_{t}\right)^{\theta}\right)\right]^{m_{t}} \theta\left(1+x_{t}\right)^{\theta-1} \exp \left(1-\left(1+x_{t}\right)^{\theta}\right)\right\}\right) \\
& \times\left[\exp \left(1-\left(1+x_{n}\right)^{\theta}\right)\right]^{k-1} \theta\left(1+x_{n}\right)^{\theta-1} \exp \left(1-\left(1+x_{n}\right)^{\theta}\right) .
\end{aligned}
$$

Theorem 2.1. Let $X$ be a continuous random variable following $\mathrm{NH}$ distribution with pdf given in (1.7), then Shannon entropy for $X$ is given as

$$
\begin{aligned}
H(\mathbf{X}(n, \tilde{m}, k))= & -n \ln \theta-\ln k-\sum_{J=1}^{n-1} \ln \gamma_{J}+\sum_{t=1}^{n-1}\left(m_{t+1}\right) \sum_{j=1}^{t} \frac{1}{\gamma_{j}}+k \sum_{j=1}^{n} \frac{1}{\gamma_{j}} \\
& +\left(\frac{\theta-1}{\theta}\right)\left\{\sum_{t=1}^{n-1} \sum_{j=1}^{t} e^{\gamma_{j}} \operatorname{Ei}\left(-\gamma_{j}\right)+\sum_{j=1}^{n} e^{\gamma_{j}} \operatorname{Ei}\left(-\gamma_{j}\right)\right\},
\end{aligned}
$$

where Ei( $\mu)$ is the exponential integral function defined as

$$
\int_{1}^{\infty} e^{-\mu x} \ln x d x=-\frac{1}{\mu} \operatorname{Ei}(-\mu)
$$


Proof. In view of (1.1) and (1.2)

$$
\begin{aligned}
H(\mathbf{X}(n, \tilde{m}, k))= & -E\left[\ln f_{\mathbf{X}(n, \tilde{m}, k)}(x)\right] \\
H(\mathbf{X}(n, \tilde{m}, k))= & -n \ln \theta-\ln k-\sum_{J=1}^{n-1} \ln \gamma_{J}-\sum_{t=1}^{n-1}\left(m_{t}+1\right)\left(1-E\left(1+x_{t}\right)^{\theta}\right) \\
& \left.-k\left(1-E\left(1+x_{n}\right)^{\theta}\right)\right)-(\theta-1) \\
& \times\left\{\sum_{t=1}^{n-1} E\left(\ln \left(1+x_{t}\right)\right)+E\left(\ln \left(1+x_{n}\right)\right)\right\} .
\end{aligned}
$$

Now in view of (1.3), we have

$$
\begin{aligned}
E\left[\left(1+x_{t}\right)^{\theta}\right]= & c_{t-1} \sum_{j=1}^{t} a_{j}(t) \int_{0}^{\infty}\left(1+x_{t}\right)^{\theta}\left[\exp \left(1-\left(1+x_{t}\right)^{\theta}\right)\right]^{\gamma_{j}-1} \\
& \times \theta\left(1+x_{t}\right)^{\theta-1} \exp \left(1-\left(1+x_{t}\right)^{\theta}\right) d x_{t} .
\end{aligned}
$$

Put $\left(1+x_{t}\right)^{\theta}=u$

$$
\begin{aligned}
E\left[\left(1+x_{t}\right)^{\theta}\right] & =c_{t-1} \sum_{j=1}^{t} a_{j}(t) \int_{1}^{\infty} u\left[e^{(1-u)}\right]^{\gamma_{j}} d u \\
& =c_{t-1} \sum_{j=1}^{t} a_{j}(t)\left[\frac{1}{\gamma_{j}}+\frac{1}{\gamma_{j}^{2}}\right] .
\end{aligned}
$$

From Balakrishnan et al. [5], we get

$$
E\left[\left(1+x_{t}\right)^{\theta}\right]=1+\sum_{j=1}^{t} \frac{1}{\gamma_{j}} .
$$

Again in view of (1.3), we have

$$
\begin{aligned}
E\left[\ln \left(\left(1+x_{t}\right)^{\theta}\right)\right]= & c_{t-1} \sum_{j=1}^{t} a_{j}(t) \int_{0}^{\infty} \ln \left[\left(1+x_{t}\right)^{\theta}\right]\left[\exp \left(1-\left(1+x_{t}\right)^{\theta}\right)\right]^{\gamma_{j}-1} \\
& \times \theta\left(1+x_{t}\right)^{\theta-1} \exp \left(1-\left(1+x_{t}\right)^{\theta}\right) d x_{t}
\end{aligned}
$$


Again by putting $\left(1+x_{t}\right)^{\theta}=u$,

$$
\begin{aligned}
E\left[\ln \left(1+x_{t}\right)\right] & =\frac{c_{t-1}}{\theta} \sum_{j=1}^{t} a_{j}(t) e^{\gamma_{j}} \int_{1}^{\infty}(\ln u) e^{\left(-\gamma_{j} u\right)} d u \\
& =\frac{c_{t-1}}{\theta} \sum_{j=1}^{t} a_{j}(t) e^{\gamma_{j}}\left[-\frac{1}{\gamma_{j}} E i\left(-\gamma_{j}\right)\right] .
\end{aligned}
$$

From Balakrishnan et al. [5], we get

$$
E\left[\ln \left(1+x_{t}\right)\right]=-\frac{1}{\theta} \sum_{j=1}^{t} e^{\gamma_{j}} \operatorname{Ei}\left(-\gamma_{j}\right)
$$

As above, we get the expressions for $E\left[\left(1+x_{n}\right)^{\theta}\right]$ and $E\left[\ln \left(1+x_{n}\right)\right]$.

Now, putting the value of $E\left[\left(1+x_{t}\right)^{\theta}\right], E\left[\ln \left(1+x_{t}\right)\right], E\left[\left(1+x_{n}\right)^{\theta}\right]$, and $E\left[\ln \left(1+x_{n}\right)\right]$ in (2.4), we get the result.

Remark 2.1. Putting $m=0, k=1$, in (2.2), we get the exact expression for Shannon entropy of $\mathrm{NH}$ distribution based on order statistics as follows:

$$
\begin{aligned}
H\left(X_{1: n}, \ldots, X_{n: n}\right)= & -n \ln \theta-\ln k-\sum_{J=1}^{n-1} \ln (n-J+1)+\sum_{t=1}^{n-1}\left(m_{t+1}\right) \sum_{j=1}^{t} \frac{1}{(n-j+1)} \\
& +k \sum_{j=1}^{n} \frac{1}{(n-j+1)}+\left(\frac{\theta-1}{\theta}\right)\left\{\sum_{t=1}^{n-1} \sum_{j=1}^{t} e^{(n-j+1)} \operatorname{Ei}(j-n-1)\right. \\
& \left.+\sum_{j=1}^{n} e^{(n-j+1)} E i(j-n-1)\right\} .
\end{aligned}
$$


Remark 2.2. Putting $m_{1}=m_{2}=\ldots=m_{n-1}=-1$ and $k \geq 1$ in (2.2), we get Shannon entropy for $k$-th record values from NH distribution as follows:

$$
H\left(X_{1}^{(k)}, \ldots, X_{n}^{(k)}\right)=-\ln (\theta k)^{n}+n+\frac{\theta-1}{\theta} e^{k} \operatorname{Ei}(-k)\left[\frac{n(n+1)}{2}\right] .
$$

\section{Single Moment and Characterization of NH Distribution Based on Generalized Order Statistics}

In this section, firstly, exact moment of gos from $\mathrm{NH}$ distribution has been deduced. Further, exact moments of order statistics, progressive type II censored order statistics and sequential order statistics are obtained as the particular cases of gos. After that applying the generalization of the Müntz-Szász theorem (Hwang and Lin [6]), we have established a characterization result of $\mathrm{NH}$ distribution based on recurrence relation of the single moment of gos.

Lemma 3.1. For any positive $(a, b)$ and $J \in \mathbb{N}$, the value of integral

$$
\begin{aligned}
\Phi^{J}(a, b) & =\int_{0}^{\infty} x^{J} e^{-a(1+x)^{b}} b(1+x)^{b-1} d x \\
& =\sum_{g=0}^{J}(-1)^{J+g}\left(\begin{array}{l}
J \\
g
\end{array}\right)\left[\frac{\Gamma\left(\frac{g}{b}+1\right)}{a^{\frac{g}{b}+1}}-\left(\frac{g}{b}+1\right)^{-1} e^{-a}{ }_{1} F_{1}\left(1 ; 1+\frac{g}{b}+1 ; a\right)\right],
\end{aligned}
$$

where ${ }_{1} F_{1}(c ; d ; z)$ is a Kummer Conuent hypergeometric function which is defined as

$$
{ }_{1} F_{1}(c ; d ; z)=\sum_{n=0}^{\infty} \frac{(c)_{n}}{(d)_{n}} \frac{z^{n}}{n !}, d \neq 0,-1,-2, \ldots
$$

and $\Gamma\left(\frac{g}{b}+1\right)$ is a gamma function defined as

$$
\Gamma\left(\frac{g}{b}+1\right)=\int_{0}^{\infty} t^{\frac{g}{b}+1-1} e^{-t} d t
$$


Proof.

$$
\Phi^{J}(a, b)=\int_{0}^{\infty} x^{J} e^{-a(1+x)^{b}} b(1+x)^{b-1} d x
$$

Put $a(1+x)^{b}=t$

$$
\begin{aligned}
a b & (1+x)^{b-1} d x=d t \\
\Phi^{J}(a, b)= & \frac{1}{a} \int_{a}^{\infty}\left[\left(\frac{t}{a}\right)^{\frac{1}{b}}-1\right]^{J} e^{-t} d t \\
= & \sum_{g=0}^{J}(-1)^{J+g} \frac{\left(\begin{array}{l}
J \\
g
\end{array}\right)}{a^{\frac{g}{b}+1}} \int_{a}^{\infty} t^{\frac{g}{b}+1-1} e^{-t} d t \\
= & \sum_{g=0}^{J}(-1)^{J+g} \frac{\left(\begin{array}{l}
J \\
g
\end{array}\right)}{a^{\frac{g}{b}+1}}\left[\Gamma\left(\frac{g}{b}+1\right)-\Upsilon\left(\frac{g}{b}+1, a\right)\right],
\end{aligned}
$$

where $\Upsilon\left(\frac{g}{b}+1, a\right)$ is a lower incomplete gamma function defined as

$$
\Upsilon\left(\frac{g}{b}+1, a\right)=\int_{0}^{\infty} t^{\frac{g}{b}+1-1} e^{-t} d t=\left(\frac{g}{b}+1\right)^{-1} a^{\frac{g}{b}+1} e^{-a}{ }_{1} F_{1}\left(1 ; 1+\frac{g}{b}+1 ; a\right) .
$$

By using (3.4), we derive the relation given in (3.1).

Theorem 3.1. Let $X$ be a non-negative continuous random variable and follows NH distribution given in (1.7), then the single moment of $r$-th gos $(1 \leq r \leq n)$, is given by

$$
\begin{aligned}
E\left[X^{J}(r, n, \tilde{m}, k)\right]= & c_{r-1} \sum_{i=1}^{r} \sum_{g=0}^{J} a_{i}(r)(-1)^{J+g}\left(\begin{array}{l}
J \\
g
\end{array}\right) \\
& \times\left[e^{\gamma_{i}} \frac{\Gamma\left(\frac{g}{\theta}+1\right)}{\gamma_{i}^{\frac{g}{\theta}+1}}-\frac{1 F_{1}\left(1 ; \frac{g}{\theta}+2 ; \gamma_{i}\right)}{\left(\frac{g}{\theta}+1\right)}\right] .
\end{aligned}
$$


Proof. In the view of (1.3), we have

$$
\begin{aligned}
E\left[X^{J}(r, n, \tilde{m}, k)\right] & =c_{r-1} \sum_{i=1}^{r} a_{i}(r) \int_{0}^{\infty} x^{J}[\bar{F}(x)]^{\gamma_{i}} \frac{f(x)}{\bar{F}(x)} d x \\
& =c_{r-1} \sum_{i=1}^{r} a_{i}(r) \int_{0}^{\infty} x^{J} e^{\left[1-(1+x)^{\theta}\right]^{\gamma_{i}}} \theta(1+x)^{\theta-1} d x \\
& =c_{r-1} \sum_{i=1}^{r} a_{i}(r) e^{\gamma_{i}} \int_{0}^{\infty} x^{J} e^{-\gamma_{i}(1+x)^{\theta}} \theta(1+x)^{\theta-1} d x .
\end{aligned}
$$

Using Lemma 3.1, we get the result.

Remark 3.1. It may be noted that for $\gamma_{i} \neq \gamma_{j}$ and $m_{1}=m_{2}=\ldots=$ $m_{n-1}=m \neq-1$,

$$
a_{i}(r)=\frac{(-1)^{r-i}}{(m+1)^{r-1}(r-1) !}\left(\begin{array}{l}
r-1 \\
i-1
\end{array}\right) \text {. }
$$

Put the value of $a_{i}(r)$ from (3.6) in (3.5), we get

$$
\begin{aligned}
E\left[X^{J}(r, n, m, k)\right]= & c_{r-1} \sum_{i=1}^{r} \sum_{g=0}^{J} \frac{(-1)^{r-i+J+g}}{(m+1)^{r-1}(r-1) !}\left(\begin{array}{l}
r-1 \\
i-1
\end{array}\right)\left(\begin{array}{l}
J \\
g
\end{array}\right) \\
& \times\left[e^{\gamma_{i}} \frac{\Gamma\left(\frac{g}{\theta}+1\right)}{\gamma_{i}^{\frac{g}{\theta}}+1}-\frac{{ }_{1} F_{1}\left(1 ; \frac{g}{\theta}+2 ; \gamma_{i}\right)}{\left(\frac{g}{\theta}+1\right)}\right] .
\end{aligned}
$$


Remark 3.2. (1) Putting $m=0, k=1$, in (3.5), the explicit formula for single moment of order statistics from $\mathrm{NH}$ distribution can be obtained as

$$
\begin{aligned}
E\left[X_{r: n}^{J}\right]= & r\left(\begin{array}{l}
n \\
r
\end{array}\right) \sum_{i=1}^{r} \sum_{g=0}^{J}(-1)^{r-i}\left(\begin{array}{l}
r-1 \\
i-1
\end{array}\right)(-1)^{J+g}\left(\begin{array}{l}
J \\
g
\end{array}\right) \\
& \times\left[e^{n-i+1} \frac{\Gamma\left(\frac{g}{\theta}+1\right)}{(n-i+1)^{\frac{g}{\theta}}+1}-\frac{{ }_{1} F_{1}\left(1 ; \frac{g}{\theta}+2 ; n-i+1\right)}{\left(\frac{g}{\theta}+1\right)}\right] .
\end{aligned}
$$

(2) Putting $n, m \in \mathbb{N}, m_{i}=R_{i}, n=m+\sum_{j=1}^{m} R_{j}, R_{j} \in \mathbb{N}_{0} \quad$ and $\gamma_{j}=n-\sum_{\nu=1}^{j-1} R_{\nu}-j+1,1 \leq j \leq m$ in (3.5), the single moment of progressive type II censored order statistics of $\mathrm{NH}$ distribution can be obtained.

(3) Putting $\gamma_{i}=(n-i+1) \beta_{i} ; \beta_{1}, \beta_{2}, \ldots, \beta_{n}>0$ in (3.5), the single moment of sequential order statistics of $\mathrm{NH}$ distribution can be obtained.

Theorem 3.2. Let $X$ be a continuous random variable, then for integer $J$ such that $J \geq 1$, the following recurrence relation is satisfied iff $X$ has cdf given in (1.8):

$$
\frac{J}{\theta \gamma_{r}} E[\phi(X(r, n, \tilde{m}, k))]=E\left[X^{J}(r, n, \tilde{m}, k)\right]-E\left[X^{J}(r-1, n, \tilde{m}, k)\right],(3.7
$$

where $\phi(x)=x^{J-1}(1+x)^{1-\theta}$ if and only if

$$
F(x)=1-\exp \left[1-(1+x)^{\theta}\right], \quad x, \theta>0 .
$$


Proof. We have

$$
\begin{aligned}
E\left[X^{J}(r, n, \tilde{m}, k)\right]= & c_{r-1} \sum_{i=1}^{r} a_{i}(r) \int_{0}^{\infty} x^{J}[\bar{F}(x)]^{\gamma_{i}} \frac{f(x)}{\bar{F}(x)} d x \\
= & c_{r-1} \int_{0}^{\infty} x^{J} f(x) \sum_{i=1}^{r} a_{i}(r)[\bar{F}(x)]^{\gamma_{i}-1} d x \\
& -c_{r-2} \int_{0}^{\infty} x^{J} f(x) \sum_{i=1}^{r-1} a_{i}(r-1)[\bar{F}(x)]^{\gamma_{i}-1} d x .
\end{aligned}
$$

Since $a_{i}(r-1)=\left(\gamma_{r}-\gamma_{i}\right) a_{i}(r)$,

$$
\begin{aligned}
E\left[X^{J}(r, n, \tilde{m}, k)\right]-E\left[X^{J}(r-1, n, \tilde{m}, k)\right] \\
=\gamma_{r} c_{r-2} \int_{0}^{\infty} x^{J} f(x)\left\{\sum_{i=1}^{r-1} a_{i}(r)[\bar{F}(x)]^{\gamma_{i}-1}+a_{r}(r)[\bar{F}(x)]^{\gamma_{r}-1}\right\} d x \\
-c_{r-2} \int_{0}^{\infty} x^{J} f(x) \sum_{i=1}^{r-1} a_{i}(r)\left(\gamma_{r}-\gamma_{i}\right)[\bar{F}(x)]^{\gamma_{i}-1} d x \\
=c_{r-2} \sum_{i=1}^{r} a_{i}(r) \int_{0}^{\infty} x^{J} \gamma_{i}[\bar{F}(x)]^{\gamma_{i}-1} f(x) d x
\end{aligned}
$$

Integrating by parts, we get

$$
\begin{aligned}
E\left[X^{J}(r, n, \tilde{m}, k)\right]- & E\left[X^{J}(r-1, n, \tilde{m}, k)\right] \\
& =\frac{J}{\theta \gamma_{r}} c_{r-1} \sum_{i=1}^{r} a_{i}(r) \int_{0}^{\infty} \phi(x)[\bar{F}(x)]^{\gamma_{i}-1} f(x) d x .
\end{aligned}
$$

So we have the result given in (3.7). 
Conversely, if the characterization condition (3.7) holds, then from (3.8), we have

$$
\begin{aligned}
E\left[X^{J}(r, n, \tilde{m}, k)\right]- & E\left[X^{J}(r-1, n, \tilde{m}, k)\right] \\
= & c_{r-2} \sum_{i=1}^{r} a_{i}(r) \int_{0}^{\infty} x^{J} \gamma_{i}[\bar{F}(x)]^{\gamma_{i}-1} f(x) d x .
\end{aligned}
$$

Thus

$$
\begin{aligned}
\frac{J}{\theta \gamma_{r}} c_{r-1} \sum_{i=1}^{r} a_{i}(r) \int_{0}^{\infty} \phi(x)[\bar{F}(x)]^{\gamma_{i}-1} f(x) d x & \\
& =\frac{c_{r-1}}{\gamma_{r}} \sum_{i=1}^{r} a_{i}(r) \int_{0}^{\infty} x^{J} \gamma_{i}[\bar{F}(x)]^{\gamma_{i}-1} f(x) d x .
\end{aligned}
$$

Integrating R.H.S. of (3.9) by parts and rearranging the terms, we get

$$
\frac{J c_{r-1}}{\gamma_{r}} \sum_{i=1}^{r} a_{i}(r) \int_{0}^{\infty}[\bar{F}(x)]^{\gamma_{i}}\left[\frac{\phi(x)}{\theta(\bar{F}(x))} f(x)-x^{J-1}\right] f(x) d x=0 .
$$

Applying a generalization of the Müntz-Szász theorem (Hwang and Lin [6]), we get

$$
\frac{\phi(x)}{\theta(\bar{F}(x))} f(x)=x^{J-1}
$$

which implies that

$$
F(x)=1-\exp \left[1-(1+x)^{\theta}\right], \quad x, \theta>0 .
$$

Hence the theorem.

\section{Product Moment and Characterization of NH Distribution Based on Generalized Order Statistics}

In this section, the product moment of gos from $\mathrm{NH}$ distribution has been deduced. Further, for the particular values of $m$ and $k$, the explicit expression for the product moment of order statistics, progressive type II 
censored order statistics and sequential order statistics are obtained as the particular cases of gos and applying the generalization of the MüntzSzász theorem (Hwang and Lin [6]), we have established a characterization result of $\mathrm{NH}$ distribution based on product moment of gos.

Lemma 4.1. For any positive $\left(a_{1}, a_{2}, b\right)$ and $J, K \in \mathbb{N}$, the value of integral

$$
\begin{aligned}
& \Phi^{J, K}\left(a_{1}, a_{2}, b\right) \\
& =\int_{0}^{\infty} \int_{x}^{\infty} x^{J} y^{K} e^{-a_{2}(1+x)^{b}} b(1+x)^{b-1} e^{-a_{1}(1+y)^{b}} b(1+y)^{b-1} d y d x \\
& =\sum_{i_{1}=0}^{K}(-1)^{K+i_{1}}\left(\begin{array}{l}
K \\
i_{1}
\end{array}\right)\left[\sum _ { i _ { 2 } = 0 } ^ { J } ( - 1 ) ^ { J + i _ { 2 } } ( \begin{array} { l } 
{ J } \\
{ i _ { 2 } }
\end{array} ) \left\{\frac { \Gamma ( \frac { i _ { 1 } } { b } + 1 ) } { a _ { 1 } ^ { \frac { i _ { 1 } } { b } + 1 } } \left\{\frac{e^{a_{2}} \Gamma\left(\frac{i_{2}}{b}+1\right)}{\left(a_{2}-a_{1}\right)^{\frac{i_{2}}{b}+1}}\right.\right.\right. \\
& \left.-\frac{e^{a_{1}}}{\left(\frac{i_{2}}{b}+1\right)}{ }_{1} F_{1}\left(1, \frac{i_{2}}{b}+1, a_{2}-a_{1}\right)\right\}-\frac{1}{\left(\frac{i_{1}}{b}+1\right)}\left\{e^{a_{2}} \frac{\Gamma \frac{i_{1}+i_{2}}{b}+2}{a_{2}^{\frac{i_{1}+i_{2}}{b}+2}}\right. \\
& \times_{2} F_{1}\left[1, \frac{i_{1}+i_{2}}{b}+2 ; \frac{i_{1}}{b}+2 ; \frac{a_{1}}{a_{2}}\right]-\frac{1}{\left(\frac{i_{1}+i_{2}}{b}+2\right)} \\
& \left.\left.\left.\times F_{1: 1 ; 0}^{0: 2 ; 1}\left(-: 1, \frac{i_{1}+i_{2}}{b}+2 ; 1 ; \frac{i_{1}+i_{2}}{b}+3: \frac{i_{1}}{b}+2 ;-; a_{1}, a_{2}\right)\right\}\right\}\right]
\end{aligned}
$$

where

$$
\begin{gathered}
{ }_{1} F_{1}(a ; b ; z)=\sum_{n=0}^{\infty} \frac{(a)_{n}}{(b)_{n}} \frac{z^{n}}{n !}, b \neq 0,-1,-2, \ldots, \\
{ }_{2} F_{1}(a ; b ; z)=\sum_{n=0}^{\infty} \frac{(a)_{n}(b)_{n}}{(c)_{n}} \frac{z^{n}}{n !}, c \neq 0,-1,-2, \ldots,
\end{gathered}
$$


(4.2) and (4.3) are known as Kummer Conuent hypergeometric function and Guass hypergeometric function, respectively.

$$
\begin{aligned}
F_{l: m ; n}^{p: q ; k}\left[\left(a_{p}\right):\left(b_{q}\right) ;\left(c_{k}\right) ;\left(\alpha_{l}\right):\left(\beta_{m}\right) ;\left(\gamma_{n}\right) ; x, y\right] \\
=\sum_{r, s=0}^{\infty} \frac{\prod_{j=1}^{p}\left(a_{j}\right)_{r+s} \prod_{j=1}^{q}\left(b_{j}\right)_{r} \prod_{j=1}^{k}\left(c_{j}\right)_{s}}{\prod_{j=1}^{l}\left(\alpha_{j}\right)_{r+s} \prod_{j=1}^{m}\left(\beta_{j}\right)_{r} \prod_{j=1}^{n}\left(\gamma_{j}\right)_{s}} \frac{x^{r}}{r !} \frac{y^{s}}{s !},
\end{aligned}
$$

is known as Kampé de Fériet function, which converges, if

(i) $p+q<l+m+1, p+k<l+n+1,|x|<\infty,|y|<\infty$,

or

(ii) $p+q=l+m+1, p+k=l+n+1,|x|<\infty,|y|<\infty$.

Proof.

$$
\begin{gathered}
\Phi^{J, K}\left(a_{1}, a_{2}, b\right)=\int_{0}^{\infty} x^{J} e^{-a_{2}(1+x)^{b}} b(1+x)^{b-1} I(x) d x, \\
I(x)=\int_{x}^{\infty} y^{K} e^{-a_{1}(1+y)^{b}} b(1+y)^{b-1} d y .
\end{gathered}
$$

Using (3.3), we get

$$
I(x)=\frac{a_{1}}{\left(e^{1-(1+x)^{b}}\right)^{a_{1}}} \sum_{i_{1}=0}^{K}(-1)^{K+i_{1}} \frac{\left(\begin{array}{l}
K \\
i_{1}
\end{array}\right)}{a_{1}^{\frac{i_{1}}{b}+1}}\left[\Gamma\left(\frac{i_{1}}{b}+1\right)-\Upsilon\left(\frac{i_{1}}{b}+1, a_{1}(1+x)^{b}\right)\right] .
$$

Putting the value of $I(x)$ in (4.5), we get

$$
\begin{aligned}
I(x)= & \sum_{i_{1}=0}^{K}(-1)^{K+i_{1}} \frac{\left(\begin{array}{l}
K \\
i_{1}
\end{array}\right)}{a_{1}^{\frac{i_{1}}{b}+1}} e^{a_{2}}\left[\int_{0}^{\infty} x^{J}\left(e^{-(1+x)^{b}}\right)^{\left(a_{2}-a_{1}\right)} b(1+x)^{b-1} \Gamma\left(\frac{i_{1}}{b}+1\right)\right. \\
& -\sum_{i_{3}=0}^{\infty} \frac{(1)_{i_{3}}}{\left(2+\frac{i_{1}}{b}\right)_{i_{3}}} \frac{a_{1}^{i_{3}}}{i_{3} !} \frac{a_{1}^{\left(\frac{i_{1}}{b}+1\right)}}{\left(\frac{i_{1}}{b}+1\right)} \int_{0}^{\infty} x^{J} e^{-a_{2}(1+x)^{b}}
\end{aligned}
$$




$$
\left.\times\left((1+x)^{b}\right)^{\frac{i_{1}}{b}+i_{3}+1} b(1+x)^{b-1}\right] d x .
$$

Again using (3.3), we get

$$
\begin{aligned}
I(x)= & \sum_{i_{1}=0}^{K}(-1)^{K+i_{1}} \frac{\left(\begin{array}{l}
K \\
i_{1}
\end{array}\right)}{a_{1}^{\frac{i_{1}}{b}+1}} e^{a_{2}}\left[\sum _ { i _ { 2 } = 0 } ^ { J } ( - 1 ) ^ { J + i _ { 2 } } ( \begin{array} { l } 
{ J } \\
{ i _ { 2 } }
\end{array} ) \left\{\frac{1}{\left(a_{2}-a_{1}\right)^{\frac{i_{2}}{b}+1}} \Gamma\left(\frac{i_{1}}{b}+1\right)\right.\right. \\
& \times\left\{\Gamma\left(\frac{i_{2}}{b}+1\right)-\Upsilon\left(\frac{i_{2}}{b}+1, a_{2}-a_{1}\right)\right\} \\
& -\sum_{i_{3}=0}^{\infty} \frac{(1)_{i_{3}}}{\left(2+\frac{i_{1}}{b}\right)_{i_{3}} a_{2}^{\frac{i_{1}+i_{2}}{b}+i_{3}+2}} \frac{a_{1}^{i_{3}}}{i_{3} !} \frac{1}{\left(\frac{i_{1}}{b}+1\right)} \\
& \left.\left.\times\left(\Gamma\left(\frac{i_{1}+i_{2}}{b}+i_{3}+2\right)-\Upsilon\left(\frac{i_{1}+i_{2}}{b}+i_{3}+2, a_{2}\right)\right)\right\}\right] .
\end{aligned}
$$

Using Lemma 3.1, we get the result given in (4.1).

Theorem 4.1. Let $X$ be a non-negative continuous random variable and follows NH distribution given in (1.4), the product moment of r-th and s-th gos is given as

$$
\begin{aligned}
& E\left[X^{J}(r, n, \tilde{m}, k) X^{K}(s, n, \tilde{m}, k)\right] \\
& =c_{s-1} \sum_{i=1}^{r} \sum_{t=r+1}^{s} \sum_{i_{1}=0}^{K} a_{i}(r) a_{t}^{(r)}(s)(-1)^{K+i_{1}}\left(\begin{array}{l}
K \\
i_{1}
\end{array}\right)\left[\sum_{i_{2}=0}^{J}(-1)^{J+i_{2}}\right. \\
& \times\left(\begin{array}{c}
J \\
i_{2}
\end{array}\right)\left\{\frac{\Gamma\left(\frac{i_{1}}{\theta}+1\right)}{\gamma_{t}\left(\frac{i_{1}}{\theta}+1\right)}\left\{\frac{e^{\gamma_{i}} \Gamma\left(\frac{i_{2}}{\theta}+1\right)}{\left(\gamma_{i}-\gamma_{t}\right)\left(\frac{i_{2}}{\theta}+1\right)}-\frac{e^{\gamma_{t}}}{\left(\frac{i_{2}}{\theta}+1\right)}{ }_{1} F_{1}\left(1 ; \frac{i_{2}}{\theta}+2 ; \gamma_{i}-\gamma_{t}\right)\right\}\right. \\
& -\frac{1}{\left(\frac{i_{1}}{\theta}+1\right)}\left\{\frac{e^{\gamma_{i}} \Gamma\left(\frac{i_{1}+i_{2}}{\theta}+2\right)}{\gamma_{i}^{\left(\frac{i_{1}+i_{2}}{\theta}+2\right)}}{ }_{2} F_{1}\left(1, \frac{i_{1}+i_{2}}{\theta}+2 ; \frac{i_{1}}{\theta}+2 ; \frac{\gamma_{t}}{\gamma_{i}}\right)\right.
\end{aligned}
$$




$$
\left.\left.\left.-\frac{1}{\left(\frac{i_{1}+i_{2}}{\theta}+2\right)} F_{1: 1 ; 0}^{0: 2 ; 1}\left(-: 1, \frac{i_{1}+i_{2}}{\theta}+2 ; 1 ; \frac{i_{1}+i_{2}}{\theta}+3: \frac{i_{1}}{\theta}+2 ;-; \gamma_{t}, \gamma_{i}\right)\right\}\right\}\right]
$$

provided that $\gamma_{1: r}>\gamma_{t}, \forall t=r+1, \ldots, s$ and $\gamma_{1: r}=\min \left(\gamma_{1}, \gamma_{2}, \ldots, \gamma_{r}\right)$.

Proof. In view of (1.4), we have

$$
\begin{aligned}
& E\left[X^{J}(r, n, \tilde{m}, k) X^{K}(s, n, \tilde{m}, k)\right] \\
& =c_{s-1} \sum_{i=1}^{r} \sum_{t=r+1}^{s} a_{i}(r) a_{t}^{(r)}(s) \int_{0}^{\infty} \int_{x}^{\infty} x^{J} y^{K}[\bar{F}(x)]^{\gamma_{i}} \frac{f(x)}{\bar{F}(x)}\left[\frac{\bar{F}(y)}{\bar{F}(x)}\right]^{\gamma_{t}} \\
& \quad \times \frac{f(y)}{\bar{F}(y)} d y d x \\
& =c_{s-1} \sum_{i=1}^{r} \sum_{t=r+1}^{s} a_{i}(r) a_{t}^{(r)}(s) \int_{0}^{\infty} \int_{x}^{\infty} x^{J} y^{K} e^{-a_{2}(1+x)^{b}} b(1+x)^{b-1} \\
& \quad \times e^{-a_{1}(1+y)^{b}} b(1+y)^{b-1} d y d x .
\end{aligned}
$$

Using Lemma 4.1, we derive the relation given in (4.6).

Remark 4.1. It may be noted that for $\gamma_{i} \neq \gamma_{t}$ and $m_{1}=m_{2}=\ldots=$ $m_{n-1}=m \neq-1$,

$$
\begin{gathered}
a_{t}^{(r)}(s)=\frac{(-1)^{s-t}}{(m+1)^{s-r-1}(s-r-1) !}\left(\begin{array}{l}
s-r-1 \\
t-r-1
\end{array}\right), \\
a_{i}(r)=\frac{(-1)^{r-i}}{(m+1)^{r-1}(r-1) !}\left(\begin{array}{l}
r-1 \\
i-1
\end{array}\right) .
\end{gathered}
$$

Put the value of $a_{t}^{(r)}(s)$ and $a_{i}(r)$ from (4.7) and (4.8) in (4.6), we get 


$$
\begin{aligned}
& E {\left[X^{J}(r, n, m, k) X^{K}(s, n, m, k)\right] } \\
&= c_{s-1} \sum_{i=1}^{r} \sum_{t=r+1}^{s} \sum_{i_{1}=0}^{K} \frac{(-1)^{s+r-i-t}}{(r-1) !(s-r-1) !(m+1)^{s-2}}\left(\begin{array}{c}
r-1 \\
i-1
\end{array}\right) \\
& \times\left(\begin{array}{l}
s-r-1 \\
t-r-1
\end{array}\right)(-1)^{K+i_{1}}\left(\begin{array}{l}
K \\
i_{1}
\end{array}\right)\left[\sum _ { i _ { 2 } = 0 } ^ { J } ( - 1 ) ^ { J + i _ { 2 } } ( \begin{array} { l } 
{ J } \\
{ i _ { 2 } }
\end{array} ) \left(\frac{\Gamma\left(\frac{i_{1}}{\theta}+1\right)}{\left(\frac{i_{1}}{\theta}+1\right)}\right.\right. \\
& \times\left\{\begin{array}{l}
e^{\gamma_{i}} \Gamma\left(\frac{i_{2}}{\theta}+1\right) \\
\left(\gamma_{i}-\gamma_{t}\right) \\
\end{array}\right. \\
&\left.-\frac{e^{\gamma_{t}}}{\theta}+\frac{\left.i_{2}\right)}{\left(\frac{i_{2}}{\theta}+1\right)}{ }_{1} F_{1}\left(1 ; \frac{i_{2}}{\theta}+2 ; \gamma_{i}-\gamma_{t}\right)\right\} \\
&-\frac{e^{\gamma_{i}} \Gamma\left(\frac{i_{1}+i_{2}}{\theta}+2\right)}{\left(\frac{i_{1}+i_{2}}{\theta}+2\right)}{ }_{2}^{\left(\frac{i_{1}+i_{2}}{\theta}+2\right)} F_{1}\left(1, \frac{i_{1}+i_{2}}{\theta}+2 ; \frac{i_{1}}{\theta}+2 ; \frac{\gamma_{t}}{\gamma_{i}}\right) \\
&\left.\left.\gamma_{i}^{0: 2 ; 1 ; 0}\left(-: 1, \frac{i_{1}+i_{2}}{\theta}+2 ; 1 ; \frac{i_{1}+i_{2}}{\theta}+3: \frac{i_{1}}{\theta}+2 ;-; \gamma_{t}, \gamma_{i}\right)\right\}\right\} .
\end{aligned}
$$

Remark 4.2. (1) Putting $m=0, k=1$, in (4.6), the explicit formula for product moment of order statistics from $\mathrm{NH}$ distribution can be obtained as

$$
\begin{aligned}
& E\left[X_{r: n}^{J} X_{s: n}^{K}\right]=\frac{n !}{(n-s) !(r-1) !(s-r-1) !} \sum_{i=1}^{r} \sum_{t=r+1}^{s} \sum_{i_{1}=0}^{K}(-1)^{K+i_{1}+s+r-i-t} \\
& \quad \times\left(\begin{array}{c}
r-1 \\
i-1
\end{array}\right)\left(\begin{array}{l}
s-r-1 \\
t-r-1
\end{array}\right)\left(\begin{array}{l}
K \\
i_{1}
\end{array}\right)\left[\sum _ { i _ { 2 } = 0 } ^ { J } ( - 1 ) ^ { J + i _ { 2 } } ( \begin{array} { l } 
{ J } \\
{ i _ { 2 } }
\end{array} ) \left\{\frac{\Gamma\left(\frac{i_{1}}{\theta}+1\right)}{(n-t+1)\left(\frac{i_{1}}{\theta}+1\right)}\right.\right.
\end{aligned}
$$




$$
\begin{aligned}
& \times\left\{\frac{e^{(n-i+1)} \Gamma\left(\frac{i_{2}}{\theta}+1\right)}{(t-i)\left(\frac{i_{2}}{\theta}+1\right)}-\frac{e^{(n-t+1)}}{\left(\frac{i_{2}}{\theta}+1\right)}{ }_{1} F_{1}\left(1 ; \frac{i_{2}}{\theta}+2 ; t-i\right)\right\} \\
& -\frac{1}{\left(\frac{i_{1}}{\theta}+1\right)}\left\{\frac{e^{(n-i+1)} \Gamma\left(\frac{i_{1}+i_{2}}{\theta}+2\right)}{(n-i+1)\left(\frac{i_{1}+i_{2}}{\theta}+2\right)}{ }_{2} F_{1}\left(1, \frac{i_{1}+i_{2}}{\theta}+2 ; \frac{i_{1}}{\theta}+2 ; \frac{n-t+1}{n-i+1}\right)\right. \\
& \left.-\frac{1}{\left(\frac{i_{1}+i_{2}}{\theta}+2\right)} F_{1: 1 ; 0}^{0: 2 ; 1}\right) \\
& \left.\left.\times\left(-: 1, \frac{i_{1}+i_{2}}{\theta}+2 ; 1 ; \frac{i_{1}+i_{2}}{\theta}+3: \frac{i_{1}}{\theta}+2 ;-;(n-t+1),(n-i+1)\right)\right\}\right\} .
\end{aligned}
$$

(2) Putting $n, m \in \mathbb{N}, m_{i}=R_{i}, n=m+\sum_{j=1}^{m} R_{j}, R_{j} \in \mathbb{N}_{0} \quad$ and $\gamma_{j}=n-\sum_{\nu=1}^{j-1} R_{\nu}-j+1,1 \leq j \leq m$, in (4.6), the product moment of progressive type II censored order statistics of $\mathrm{NH}$ distribution can be obtained.

(3) Putting $\gamma_{i}=(n-i+1) \beta_{i} ; \beta_{1}, \beta_{2}, \ldots, \beta_{n}>0$ in (4.6), the product moment of sequential order statistics of $\mathrm{NH}$ distribution can be obtained.

Theorem 4.2. Let $X$ be a random variable, then for integers $J$ and $K$ such that $J, K \geq 1$, the following recurrence relation is satisfied iff $X$ has $c d f(1.8)$ :

$$
\begin{aligned}
\frac{K}{\theta \gamma_{s}} E[\phi(X(r, n, \tilde{m}, k) X(s, n, \tilde{m}, k))]= & E\left[X^{J}(r, n, \tilde{m}, k) X^{K}(s, n, \tilde{m}, k)\right] \\
& -E\left[X^{J}(r, n, \tilde{m}, k) X^{K}(s-1, n, \tilde{m}, k)\right],
\end{aligned}
$$


where $\phi(x, y)=x^{J} y^{K-1}(1+y)^{1-\theta}$ if and only if

$$
F(y)=1-\exp \left[1-(1+y)^{\theta}\right], \quad y, \theta>0 .
$$

Proof. Using (1.4) and (4.9), we get

$$
\begin{aligned}
& E\left[X^{J}(r, n, \tilde{m}, k) X^{K}(s, n, \tilde{m}, k)\right]-E\left[X^{J}(r, n, \tilde{m}, k) X^{K}(s-1, n, \tilde{m}, k)\right] \\
= & c_{s-1} \int_{0}^{\infty} \int_{x}^{\infty} x^{J} y^{K} \sum_{t=r+1}^{s} a_{t}^{(r)}(s)\left[\frac{\bar{F}(y)}{\bar{F}(x)}\right]^{\gamma_{t}}\left[\sum_{i=1}^{r} a_{i}(r)[\bar{F}(x)]^{\gamma_{i}}\right] \frac{f(x)}{\bar{F}(x)} \times \frac{f(y)}{\bar{F}(y)} d y d x \\
- & c_{s-2} \int_{0}^{\infty} \int_{x}^{\infty} x^{J} y^{K} \sum_{t=r+1}^{s-1} a_{t}^{(r)}(s-1)\left[\frac{\bar{F}(y)}{\bar{F}(x)}\right]^{\gamma_{t}}\left[\sum_{i=1}^{r} a_{i}(r)[\bar{F}(x)]^{\gamma_{i}}\right] \frac{f(x)}{\bar{F}(x)} \times \frac{f(y)}{\bar{F}(y)} d y d x .
\end{aligned}
$$

Since $a_{t}^{(r)}(s-1)=\left(\gamma_{s}-\gamma_{i}\right) a_{t}^{(r)}(s)$,

$$
\begin{aligned}
& E\left[X^{J}(r, n, \tilde{m}, k) X^{K}(s, n, \tilde{m}, k)\right]-E\left[X^{J}(r, n, \tilde{m}, k) X^{K}(s-1, n, \tilde{m}, k)\right] \\
& =c_{s-2} \int_{0}^{\infty} \int_{x}^{\infty} x^{J} y^{K}\left\{\sum_{t=r+1}^{s-1} a_{t}^{(r)}(s) \gamma_{t}\left[\frac{\bar{F}(y)}{\bar{F}(x)}\right]^{\gamma_{t}}\right. \\
& \left.+\gamma_{s} a_{s}^{(r)}(s)\left[\frac{\bar{F}(y)}{\bar{F}(x)}\right]^{\gamma_{s}}\right\}\left[\sum_{i=1}^{r} a_{i}(r)[\bar{F}(x)]^{\gamma_{i}}\right] \frac{f(x)}{\bar{F}(x)} \times \frac{f(y)}{\bar{F}(y)} d y d x \\
& =c_{s-2} \int_{0}^{\infty} x^{J} \sum_{i=1}^{r} a_{i}(r)[\bar{F}(x)]^{\gamma_{i}} \frac{f(x)}{\bar{F}(x)} \int_{x}^{\infty} y^{K}\left\{\sum_{t=r+1}^{s} a_{t}^{(r)}(s) \gamma_{t}\left[\frac{\bar{F}(y)}{\bar{F}(x)}\right]^{\gamma_{t}} \times \frac{f(y)}{\bar{F}(y)} d y\right\} d x .
\end{aligned}
$$

Now integrating the second integral of (4.11) by parts, we get

$$
\begin{aligned}
& E\left[X^{J}(r, n, \tilde{m}, k) X^{K}(s, n, \tilde{m}, k)\right]-E\left[X^{J}(r, n, \tilde{m}, k) X^{K}(s-1, n, \tilde{m}, k)\right] \\
& =\frac{K}{\theta \gamma_{s}} c_{s-1} \int_{0}^{\infty} \phi(x, y) \sum_{i=1}^{r} a_{i}(r)[\bar{F}(x)]^{\gamma_{i}} \frac{f(x)}{\bar{F}(x)} \int_{x}^{\infty}\left\{\sum_{t=r+1}^{s} a_{t}^{(r)}(s)\left[\frac{\bar{F}(y)}{\bar{F}(x)}\right]^{\gamma_{t}} \times \frac{f(y)}{\bar{F}(y)} d y\right\} d x .
\end{aligned}
$$


Hence, we get the relation given in (4.9).

Conversely, if the characterizing condition (4.9) holds then from (4.12), we have

$$
\begin{aligned}
& \frac{K}{\theta \gamma_{s}} c_{s-1} \int_{0}^{\infty} \phi(x, y) \sum_{i=1}^{r} a_{i}(r)[\bar{F}(x)]^{\gamma_{i}} \frac{f(x)}{\bar{F}(x)} \int_{x}^{\infty}\left\{\sum_{t=r+1}^{s} a_{t}^{(r)}(s)\left[\frac{\bar{F}(y)}{\bar{F}(x)}\right]^{\gamma_{t}} \times \frac{f(y)}{\bar{F}(y)} d y\right\} d x \\
& =c_{s-1} \int_{0}^{\infty} \int_{x}^{\infty} x^{J} y^{K} \sum_{t=r+1}^{s} a_{t}^{(r)}(s)\left[\frac{\bar{F}(y)}{\bar{F}(x)}\right]^{\gamma_{t}}\left[\sum_{i=1}^{r} a_{i}(r)[\bar{F}(x)]^{\gamma_{i}}\right] \frac{f(x)}{\bar{F}(x)} \times \frac{f(y)}{\bar{F}(y)} d y d x \\
& -c_{s-2} \int_{0}^{\infty} \int_{x}^{\infty} x^{J} y^{K} \sum_{t=r+1}^{s-1} a_{t}^{(r)}(s-1)\left[\frac{\bar{F}(y)}{\bar{F}(x)}\right]^{\gamma_{t}}\left[\sum_{i=1}^{r} a_{i}(r)[\bar{F}(x)]^{\gamma_{i}}\right] \frac{f(x)}{\bar{F}(x)} \times \frac{f(y)}{\bar{F}(y)} d y d x \\
& =c_{s-2} \int_{0}^{\infty} x^{J} \sum_{i=1}^{r} a_{i}(r)[\bar{F}(x)]^{\gamma_{i}} \frac{f(x)}{\bar{F}(x)} \int_{x}^{\infty} y^{K}\left\{\sum_{t=r+1}^{s} a_{t}^{(r)}(s) \gamma_{t}\left[\frac{\bar{F}(y)}{\bar{F}(x)}\right]^{\gamma_{t}} \times \frac{f(y)}{\bar{F}(y)} d y\right\} d x .
\end{aligned}
$$

Now integrating the second integral of (4.13) by parts,

$$
\begin{aligned}
& \frac{K}{\theta \gamma_{s}} c_{s-1} \int_{0}^{\infty} \phi(x, y) \sum_{i=1}^{r} a_{i}(r)[\bar{F}(x)]^{\gamma_{i}} \frac{f(x)}{\bar{F}(x)} \int_{x}^{\infty}\left\{\sum_{t=r+1}^{s} a_{t}^{(r)}(s)\left[\frac{\bar{F}(y)}{\bar{F}(x)}\right]^{\gamma_{t}} \times \frac{f(y)}{\bar{F}(y)} d y\right\} d x \\
& =\frac{c_{s-1}}{\gamma_{s}} \int_{0}^{\infty} x^{J} \sum_{i=1}^{r} a_{i}(r)[\bar{F}(x)]^{\gamma_{i}} \frac{f(x)}{\bar{F}(x)} \int_{x}^{\infty} K y^{K-1}\left\{\sum_{t=r+1}^{s} a_{t}^{(r)}(s)\left[\frac{\bar{F}(y)}{\bar{F}(x)}\right]^{\gamma_{t}} d y\right\} d x,
\end{aligned}
$$

which reduces to

$$
\begin{aligned}
& \frac{c_{s-1}}{\gamma_{s}} \int_{0}^{\infty} x^{J} \sum_{i=1}^{r} a_{i}(r)[\bar{F}(x)]^{\gamma_{i}} \frac{f(x)}{\bar{F}(x)} \\
& \quad \times \int_{x}^{\infty} K\left\{\sum_{t=r+1}^{s} a_{t}^{(r)}(s)\left[\frac{\bar{F}(y)}{\bar{F}(x)}\right]^{\gamma_{t}}\left[\frac{\phi(x, y)}{\theta} \frac{f(y)}{\bar{F}(y)}-x^{J} y^{K-1}\right] d y\right\} d x=0 .
\end{aligned}
$$


Applying a generalization of the Müntz-Szász theorem (Hwang and Lin [6]), we get

$$
\frac{\phi(x, y)}{\theta} \frac{f(y)}{\bar{F}(y)}-x^{J} y^{K-1}
$$

which implies that

$$
F(y)=1-\exp \left[1-(1+y)^{\theta}\right], \quad y, \theta>0 .
$$

\section{Numerical Computations}

In this section, we have computed the values of mean and variance for order statistics and gos for $\theta=2$ using MATLAB and these are presented in Tables $1 \& 2$ and $3 \& 4$, respectively.

From Tables 1 and 2, it can be seen that the value of mean and variance decrease as the sample size increases and increase as the value of $r$ increases.

Table 1. Mean for the $r$-th order statistics from NH distribution for $\theta=2$

\begin{tabular}{|c|c|c|c|c|c|c|c|c|}
\hline$n$ & $r=1$ & $r=2$ & $r=3$ & $r=4$ & $r=5$ & $r=6$ & $r=7$ & $r=8$ \\
\hline 1 & 0.3789 & & & & & & & \\
2 & 0.2107 & 0.5472 & & & & & & \\
3 & 0.147 & 0.338 & 0.6518 & & & & & \\
4 & 0.1132 & 0.2486 & 0.4274 & 0.7266 & & & & \\
5 & 0.0921 & 0.1975 & 0.3252 & 0.4956 & 0.7843 & & & \\
6 & 0.0777 & 0.1642 & 0.2642 & 0.3861 & 0.5504 & 0.8311 & & \\
7 & 0.0672 & 0.1407 & 0.2231 & 0.3189 & 0.4365 & 0.5959 & 0.8703 & \\
8 & 0.0592 & 0.1231 & 0.1934 & 0.2727 & 0.3652 & 0.4793 & 0.6348 & 0.9039 \\
\hline
\end{tabular}


Table 2. Variance for the $r$-th order statistics from NH distribution for $\theta=2$

\begin{tabular}{|c|c|c|c|c|c|c|c|c|}
\hline$n$ & $r=1$ & $r=2$ & $r=3$ & $r=4$ & $r=5$ & $r=6$ & $r=7$ & $r=8$ \\
\hline 1 & 0.0985 & & & & & & & \\
2 & 0.0342 & 0.1062 & & & & & & \\
3 & 0.0177 & 0.0431 & 0.105 & & & & & \\
4 & 0.0109 & 0.0244 & 0.0457 & 0.1023 & & & & \\
5 & 0.0074 & 0.0159 & 0.0273 & 0.0464 & 0.0997 & & & \\
6 & 0.0053 & 0.0113 & 0.0185 & 0.0286 & 0.0463 & 0.0972 & & \\
7 & 0.004 & 0.0084 & 0.0135 & 0.02 & 0.0292 & 0.0459 & 0.095 & \\
8 & 0.0032 & 0.0066 & 0.0104 & 0.0149 & 0.0208 & 0.0294 & 0.0453 & 0.093 \\
\hline
\end{tabular}

Now Tables 3 and 4 provide the mean and variance of gos which increase and decrease as the value of $r$ and sample size increase, respectively.

Table 3. Mean for the $r$-th generalized order statistics from $\mathrm{NH}$ distribution for $\theta=2, m=2$, and $k=2$

\begin{tabular}{|c|c|c|c|c|c|c|c|c|}
\hline$n$ & $r=1$ & $r=2$ & $r=3$ & $r=4$ & $r=5$ & $r=6$ & $r=7$ & $r=8$ \\
\hline 1 & 0.2107 & & & & & & & \\
2 & 0.0921 & 0.2898 & & & & & & \\
3 & 0.0592 & 0.1469 & 0.3374 & & & & & \\
4 & 0.0436 & 0.1006 & 0.1855 & 0.3711 & & & & \\
5 & 0.0346 & 0.0769 & 0.1323 & 0.2151 & 0.3971 & & & \\
6 & 0.0286 & 0.0623 & 0.1036 & 0.1578 & 0.239 & 0.4182 & & \\
7 & 0.0244 & 0.0524 & 0.0854 & 0.1258 & 0.1791 & 0.259 & 0.4358 & \\
8 & 0.0213 & 0.0452 & 0.0727 & 0.1051 & 0.1449 & 0.1973 & 0.2762 & 0.4511 \\
\hline
\end{tabular}


Table 4. Variance for the $r$-th generalized order statistics from $\mathrm{NH}$ distribution for $\theta=2, m=2$, and $k=2$

\begin{tabular}{|c|c|c|c|c|c|c|c|c|}
\hline$n$ & $r=1$ & $r=2$ & $r=3$ & $r=4$ & $r=5$ & $r=6$ & $r=7$ & $r=8$ \\
\hline 1 & 0.0342 & & & & & & & \\
2 & 0.0074 & 0.0365 & & & & & & \\
3 & 0.0032 & 0.0095 & 0.0365 & & & & & \\
4 & 0.0018 & 0.0046 & 0.0104 & 0.036 & & & & \\
5 & 0.0011 & 0.0027 & 0.0053 & 0.0108 & 0.0355 & & & \\
6 & 0.000777 & 0.0018 & 0.0033 & 0.0057 & 0.0109 & 0.035 & & \\
7 & 0.00057 & 0.0013 & 0.0022 & 0.0036 & 0.006 & 0.011 & 0.0345 & \\
8 & 0.000435 & 0.000973 & 0.0016 & 0.0026 & 0.0038 & 0.0061 & 0.011 & 0.0341 \\
\hline
\end{tabular}

\section{Conclusion}

In this paper, we have derived the exact and analytical expression for Shannon entropy of NH distribution based on gos and its special cases such as order statistics and record values. Characterization based on recurrence relation for single and product moment of gos from $\mathrm{NH}$ distribution are also deduced. We have used this expression of single moment for calculating the means and variances from $\mathrm{NH}$ distribution.

\section{References}

[1] B. Afhami and M. Madadi, Shannon entropy in generalized order statistics from Pareto-type distributions, Int. J. Nonlinear Anal. Appl. 4(1) (2013), 79-91.

[2] A. A. Ahmad, Single and product moments of generalized order statistics from linear exponential distribution, Comm. Statist.-Theory Methods 37(8) (2008), 1162-1172.

DOI: https://doi.org/10.1080/03610920701713344

[3] M. Ahsanullah, Generalized order statistics from exponential distribution, J. Statist. Plann. Inference 85(1-2) (2000), 85-91.

DOI: https://doi.org/10.1016/S0378-3758(99)00068-3

[4] G. Arslan, On a characterization of the uniform distribution by generalized order statistics, J. Comput. Appl. Math. 235(16) (2011), 4532-4536.

DOI: https://doi.org/10.1016/j.cam.2010.02.040 
[5] N. Balakrishnan, E. Cramer and U. Kamps, Bounds for means and variances of progressive type II censored order statistics, Statist. Probab. Lett. 54(3) (2001), 301-315.

DOI: https://doi.org/10.1016/S0167-7152(01)00104-3

[6] J. S. Hwang and G. D. Lin, On a generalized moments problem II, Proc. Amer. Math. Soc. 91(4) (1984), 577-580.

DOI: https://doi.org/10.1090/S0002-9939-1984-0746093-4

[7] U. Kamps, A Concept of Generalized Order Statistics, Teubner, Stuttgart, 1995.

[8] U. Kamps, Characterizations of Distributions by Recurrence Relations and Identities for Moments of Order Statistics, In: N. Balakrishnan and C. R. Rao, Editors, Handbook of Statistics, 16, Order Statistics: Theory and Methods, Amsterdam, North-Holland (1998), 291-311.

[9] U. Kamps and E. Cramer, On distributions of generalized order statistics, Statistics 35(3) (2001), 269-280.

DOI: https://doi.org/10.1080/02331880108802736

[10] A. H. Khan and M. J. S. Khan, On ratio and inverse moments of generalized order statistics from Burr distribution, Pakistan J. Statist. 28(1) (2012), 59-68.

[11] R. U. Khan and D. Kumar, On moments of generalized order statistics from exponentiated Pareto distribution and its characterization, Appl. Math. Sci. (Ruse) 4(55) (2010), 2711-2722.

[12] A. Kulshrestha, R. U. Khan and D. Kumar, On moment generating function of generalized order statistics from Erlang-Truncated exponential distribution, Open J. Statist. 2(5) (2012), 557-564.

DOI: https://doi.org/10.4236/ojs.2012.25071

[13] D. Kumar, Generalized order statistics from Kumaraswamy distribution and its characterization, Tamsui Oxford Journal of Information and Mathematical Sciences 27(4) (2011), 463-476.

[14] D. Kumar and S. Dey, Relations for moments of generalized order statistics from extended exponential distribution, Amer. J. Math. Management Sci. 36(4) (2017), 378-400.

DOI: https://doi.org/10.1080/01966324.2017.1369474

[15] D. Kumar, S. Dey and S. Nadarajah, Extended exponential distribution based on order statistics, Comm. Statist.-Theory Methods 46(18) (2017), 9166-9184.

DOI: https://doi.org/10.1080/03610926.2016.1205625

[16] A. J. Lemonte, A new exponential-type distribution with constant, decreasing, increasing, upside-down bathtub and bathtub-shaped failure rate function, Comput. Statist. Data Anal. 62 (2013), 149-170.

DOI: https://doi.org/10.1016/j.csda.2013.01.011 
[17] M. Madadi and M. Tata, The Rényi information in record data from an inverse sampling plan, General Mathematics 19(4) (2011), 19-36.

[18] M. Madadi and M. Tata, Shannon information in record data, Metrika 74(1) (2011), 11-31.

DOI: https://doi.org/10.1007/s00184-009-0287-7

[19] M. Madadi and M. Tata, Shannon information in $k$-records, Comm. Statist.-Theory Methods 43(15) (2014), 3286-3301.

DOI: https://doi.org/10.1080/03610926.2012.697965

[20] M. R. Mahmoud and A. S. Abd El Ghafour, Shannon entropy for the generalized Feller-Pareto (GFP) family and order statistics of GFP subfamilies, Appl. Math. Sci. 7(65) (2013), 3247-3253.

DOI: http://dx.doi.org/10.12988/ams.2013.3296

[21] S. M. T. K. MirMostafaee, A. Asgharzadeh and A. Fallah, Record values from NH distribution and associated inference, Metron 74(1) (2016), 37-59.

DOI: https://doi.org/10.1007/s40300-015-0069-0

[22] S. Nadarajah and F. Haghighi, An extension of the exponential distribution, Statistics 45(6) (2011), 543-558.

DOI: https://doi.org/10.1080/02331881003678678

[23] M. A. Selim, Estimation and prediction for Nadarajah-Haghighi distribution based on record values, Pakistan J. Statist. 34(1) (2018), 77-90.

[24] C. E. Shannon, A mathematical theory of communication, Bell System Technical Journal 27(3) (1948), 379-423.

DOI: https://doi.org/10.1002/j.1538-7305.1948.tb01338.x

[25] J. C. Su and W. J. Huang, Characterizations based on conditional expectations, Statist. Papers 41(4) (2000), 423-435.

DOI: https://doi.org/10.1007/BF02925761

[26] K. M. Wong and S. Chen, The entropy of ordered sequences and order statistics, IEEE Transactions of Information Theory 36(2) (1990), 276-284.

DOI: https://doi.org/10.1109/18.52473 